\title{
ON THE BEHAVIOR OF FOURIER COEFFICIENTS
}

R. MOHANTY AND M. NANDA

1. Let $f(t)$ be integrable $L$ in $(-\pi, \pi)$ and periodic with period $2 \pi$, and let

(1) $f(t) \sim \frac{1}{2} a_{0}+\sum_{1}^{\infty}\left(a_{n} \cos n t+b_{n} \sin n t\right)=\frac{1}{2} a_{0}+\sum_{1}^{\infty} A_{n}(t)$.

Then the conjugate series of (1) at $t=x$ is

$$
\sum_{1}^{\infty}\left(b_{n} \cos n x-a_{n} \sin n x\right)=\sum_{1}^{\infty} B_{n}(x)
$$

We write

(3) $\theta(t)=f(x+t)-f(x-t), \quad \psi(t)=f(x+t)-f(x-t)-l$, where $l$ is a finite number.

It is known ${ }^{1}$ that if $\psi(t) \rightarrow 0$ as $t \rightarrow 0$, then the sequence $\{n B n(x)\}$ is summable $(C, r), r>1$, to the value $l / \pi$, but not necessarily summable $(C, 1)$. It is also known ${ }^{2}$ that with the same hypothesis the sequence $\{n B n(x)\}$ is summable by the first logarithmic mean to the value $l / \pi$.

The object of this note is to prove the following

TheOREM 1. If

$$
\psi(t)=o\left\{\left(\log \frac{1}{t}\right)^{-1}\right\}
$$

and

$$
a_{n}=O\left(n^{-\delta}\right), \quad b_{n}=O\left(n^{-\delta}\right), \quad 0<\delta<1,
$$

then the sequence $\{n B n(x)\}$ is summable $(C, 1)$ to the value $l / \pi$.

2. Proof of the theorem. We are to prove that

$$
\frac{1}{n} \sum_{1}^{n} r B_{r}(x) \rightarrow \frac{l}{\pi} \quad \text { as } n \rightarrow \infty .
$$

Writing $t_{n}(x)=\sum_{1}^{n} r B_{r}(x)$, we have

Received by the editors November 19, 1952 and, in revised form, June 30, 1953.

1 See Zygmund, Trigonometrical series, 1935, p. 62.

Zygmund, loc. cit. 


$$
\frac{t_{n}(x)}{n}=\frac{1}{\pi} \int_{0}^{\pi}\{f(x+t)-f(x-t)\} g(n, t) d t
$$

where

$$
g(n, t)=\frac{1}{n} \sum_{1}^{n} r \sin r t
$$

Denoting $\int_{0}^{\pi} g(n, t) d t$ by $\lambda_{n}$, we have

$$
\lambda_{n}=\frac{1}{n} \sum_{1}^{n}\left\{1-(-1)^{r}\right\}=1+o(1), \quad \text { as } n \rightarrow \infty .
$$

Therefore

$$
\begin{aligned}
\frac{1}{n} t_{n}(x)-\frac{l}{\pi} \lambda_{n} & =\frac{1}{\pi} \int_{0}^{\pi}\{f(x+t)-f(x-t)-l\} g(n, t) d t \\
& =\frac{1}{\pi} \int_{0}^{\pi} \psi(t) g(n, t) d t .
\end{aligned}
$$

Thus

$$
\begin{aligned}
\frac{1}{n} t_{n}(x)-\frac{l}{\pi} & =\frac{1}{\pi} \int_{0}^{\pi} \psi(t) g(n, t) d t+o(1) \\
& =J+o(1), \text { say. }
\end{aligned}
$$

We have

$$
\begin{aligned}
g(n, t) & =-\frac{1}{n} \frac{d}{d t}\{1 / 2+\cos t+\cos 2 t+\cdots+\cos n t\} \\
& =-\frac{1}{n} \frac{d}{d t}\left\{\frac{\sin (n+1 / 2) t}{2 \sin (t / 2)}\right\} \\
& =\frac{1}{4 n} \frac{\sin (n+1 / 2) t \cos (t / 2)}{\sin ^{2}(t / 2)}-\frac{(n+1 / 2)}{2 n} \frac{\cos (n+1 / 2) t}{\sin (t / 2)} .
\end{aligned}
$$

Let

(7) $J=\int_{0}^{\pi} \psi(t) g(n, t) d t=\int_{0}^{n^{-}}+\int_{n^{-1}}^{n^{-r}}+\int_{n^{-r}}^{\pi}=J_{1}+J_{2}+J_{3}$,

where $r=\delta / 2$. We require the following inequalities for $g(n, t)$.

$$
g(n, t)=O\left(n^{2} t\right),
$$

$$
g(n, t)=O\left(t^{-1}\right) \text {. }
$$


ProOF OF (8).

$g(n, t)=\frac{\sin (n+1 / 2) t \cos (t / 2)-2(n+1 / 2) \cos (n+1 / 2) t \sin (t / 2)}{4 n \sin ^{2}(t / 2)}$.

By replacing the sines and cosines in the numerator of the above fraction by their respective power series expansions it is easy to see that, for $0<t<1 / n$, the numerator is $O\left(n^{3} t^{3}\right)$ and hence

$$
|g(n, t)|<K\left(n^{3} t^{3} / n t^{2}\right)=K n^{2} t,
$$

which completes the proof of (8).

Proof of (9). Now

$$
\left|\frac{\sin (n+1 / 2) t \cos (t / 2)}{4 n \sin ^{2}(t / 2)}\right|<\frac{(n+1 / 2) t \pi^{2}}{4 n t^{2}}<\frac{\pi^{2}}{2} t^{-1} .
$$

Similarly

$$
\left|\frac{-(n+1 / 2) \cos (n+1 / 2) t}{2 n \sin (t / 2)}\right|<\pi t^{-1}
$$

Therefore,

$$
|g(n, t)|<\left(\pi+\pi^{2} / 2\right) t^{-1},
$$

which completes the proof of (9).

Using (8) we find

$$
J_{1}=\int_{0}^{n^{-1}} \psi(t) g(n, t) d t=\int_{0}^{n^{-1}} o(1) O\left(n^{2} t\right) d t=o(1) .
$$

By (9) we have

$$
\left|J_{2}\right|=o\left\{\int_{n^{-1}}^{n^{-r}} \frac{d t}{t \log (1 / t)}\right\}=o(1) .
$$

Now we write

$$
J_{3}=J_{3.1}-J_{3.2}
$$

where

$$
\begin{gathered}
J_{3.1}=\frac{1}{4 n} \int_{n-}^{\pi} \psi(t) \frac{\cos (t / 2) \sin (n+1 / 2) t}{\sin ^{2}(t / 2)} d t, \\
J_{3.2}=\frac{n+1 / 2}{2 n} \int_{n-}^{\pi} \psi(t) \frac{\cos (n+1 / 2) t}{\sin (t / 2)} d t .
\end{gathered}
$$


We take up $J_{3.2}$ first. Now the Fourier series for $\psi(t)$ is

$$
2 \sum_{1}^{\infty} B_{n}(x) \sin n t-l
$$

By multiplying the Fourier series of $\psi(t)$ by $\cos (n+1 / 2) t / \sin (t / 2)$ which is of bounded variation and integrating term by term, we have ${ }^{3}$

$$
\begin{aligned}
\int_{n \top}^{\pi} \psi(t) \frac{\cos (n+1 / 2) t}{\sin (t / 2)} d t= & \sum_{k=1}^{\infty} B_{k} \int_{n \top}^{\pi} \frac{2 \sin k t \cos (n+1 / 2) t}{\sin (t / 2)} d t \\
& -l \int_{n}^{\pi} \frac{\cos (n+1 / 2) t}{\sin (t / 2)} d t .
\end{aligned}
$$

Now the coefficient of $B_{k}$ equals

$$
\begin{aligned}
& \int_{n^{-r}}^{\pi} \frac{\sin (n+1 / 2+k) t-\sin (n+1 / 2-k) t}{\sin (t / 2)} d t \\
& \quad=\left(\operatorname{cosec}\left(n^{-r} / 2\right)\right) \int_{n^{-r}}^{\xi}\{\sin (n+1 / 2+k) t-\sin (n+1 / 2-k) t\} d t \\
& \quad \quad\left(n^{-r}<\xi<\pi\right)
\end{aligned}
$$

and it follows that the coefficient of $B_{k}$ does not exceed $4 \pi n^{r} /|k-(n+1 / 2)|$ in absolute value.

By the second mean value theorem, we find

$$
\int_{n \rightarrow}^{\pi} \frac{\cos (n+1 / 2) t}{\sin (t / 2)} d t=o(1)
$$

Thus, by (14),

Now

$$
\begin{aligned}
\left|J_{3.2}\right| & <o(1)+4 \pi \sum_{k=1}^{\infty} \frac{k^{-\delta} n^{r}}{|k-(n+1 / 2)|}=o(1)+\sum_{k=1}^{n}+\sum_{k=n+1}^{\infty} \\
& \equiv o(1)+R_{1}+R_{2} .
\end{aligned}
$$

$$
\begin{aligned}
\frac{R_{1}}{4 \pi} & <\frac{n^{r}}{(n+1) / 2} \sum_{1 \leqq k \leqq n / 2} k^{-\delta}+n^{r}\left(\frac{n}{2}\right)^{-\delta} \sum_{n / 2 \leqq k \leqq n} \frac{1}{(n+1 / 2)-k} \\
& =O\left(n^{-\delta / 2}\right)+O\left(n^{-\delta / 2} \log n\right)=o(1),
\end{aligned}
$$

and

$$
\begin{aligned}
\frac{R_{2}}{4 \pi} & <n^{r} n^{-\delta} \sum_{n<k \leqq 2 n} \frac{1}{k-(n+1 / 2)}+2 n^{r} \sum_{k>2 n} \frac{k^{-\delta}}{k-1} \\
& =O\left(n^{-\delta / 2} \log n\right)+O\left(n^{-\delta / 2}\right)=o(1) .
\end{aligned}
$$

${ }^{3}$ Zygmund, Trigonometrical series, 1935. The discussion of $J_{3.2}$ is effected by arguments similar to those in Zygmund and has been given only for the sake of completeness. 


\section{Hence}

$$
J_{3.2}=o(1) \text {. }
$$

Again

$$
J_{3.1}=\frac{1}{2 n} \int_{n \rightarrow r}^{\pi} \frac{\psi(t)\{\sin (n+1) t+\sin n t\} d t}{\sin ^{2}(t / 2)}=I_{3}+I_{4},
$$

where

$$
I_{8}=\frac{1}{2 n} \int_{n \rightarrow r}^{\pi} \psi(t) \frac{\sin (n+1) t}{\sin ^{2}(t / 2)} d t
$$

and

$$
I_{4}=\frac{1}{2 n} \int_{n-}^{\pi} \psi(t) \frac{\sin n t}{\sin ^{2}(t / 2)} d t .
$$

By applying arguments similar to that used above it can be easily shown that

$$
I_{8}=O\left(\frac{\log n}{n}\right)=o(1)
$$

and

$$
I_{4}=O\left(\frac{\log n}{n}\right)=o(1)
$$

Hence

$$
J_{3.1}=o(1) \text {. }
$$

Therefore by (12), (18), and (15) we have

$$
J_{3}=o(1) \text {. }
$$

By (7), (10), (11), and (19), we have

$$
J=o(1) \text {. }
$$

Therefore by (6) and (20),

$$
\frac{1}{n} t_{n}(x)-\frac{l}{\pi}=o(1) \quad \text { as } n \rightarrow \infty \text {, }
$$

and this completes the proof of the theorem.

3. It is easy to see from Schwarz's example of a divergent Fourier series at a point of continuity slightly modified that condition (4) 
alone does not imply summability $(C, 1)$ of the sequence $n B_{n}(x)$.

Hardy-Littlewood's convergence criterion for the allied series is ${ }^{4}$

TheOREM A. If

$$
\theta(t)=o\left\{\left(\log \frac{1}{t}\right)^{-1}\right\}
$$

and

A(ii)

$$
\text { each of } a_{n} \text { and } b_{n} \text { is } O\left(n^{-\delta}\right) \text {, }
$$$$
0<\delta<1
$$

then the allied series $\sum_{1}^{\infty} B_{n}(x)$ converges to the value

$$
\frac{1}{2 \pi} \int_{\rightarrow 0}^{\pi} \theta(t) \cot (t / 2) d t
$$

provided that the integral exists as a "Cauchy integral" at the origin.

We shall now deduce Theorem A as a corollary of our theorem by employing the following known result also due to Hardy and Littlewood. ${ }^{5}$

THEOREM B. If $\sum u_{n}$ is summable (A), then a necessary and sufficient condition that it should be summable $(C, k), k)>-1$, is that the sequence $\left\{n u_{n}\right\}$ is summable $(C, k+1)$ to the value 0 .

Proof of Theorem A. The existence of the integral (21) as a "Cauchy integral" at the origin implies the summability (A) of the allied series $\sum_{1}^{\infty} B_{n}(x) .^{6}$

By using Theorem 1, we find that conditions $A(i)$ and $A(i i)$ of Theorem A imply the summability $(C, 1)$ of the sequence $\left\{n B_{n}(x)\right\}$ to the value 0 . Now the convergence of the allied series is a consequence of Theorem $\mathrm{B}$.

Ravenshaw College, Cuttack, India

4 Hardy and Littlewood, Annali della R. Scuola Normale Superiori di Pisa (2) vol. 3 (1934) pp. 43-62, Theorem 13. See also Zygmund, Trigonometrical series, 1935, p. 35, where the slightly stronger theorem, viz.: If (i) $f(x+h)-f(x)=O(\log 1 /|h|)^{-1}$, and (ii) each of $a_{n}$ and $b_{n}$ is $O\left(n^{-\delta}\right), \delta>0$, then $\bar{S}_{n}(x)-\bar{f}(x, \pi / n)=o(1)$ (in the notation of $Z$ ygmund), is given, which includes Theorem A.

- Hardy and Littlewood, J. London Math. Soc. vol. 6 (1931) p. 283.

- Zygmund, Trigonometrical series, 1935, p. 55. 